Journal of Low Temperature Physics manuscript No.

(will be inserted by the editor)

Yu.M. Bunkov ${ }^{1}$ and G.E. Volovik ${ }^{2,3}$

\title{
Bose-Einstein condensation of magnons in superfluid ${ }^{3} \mathrm{He}$
}

XX.XX.2007

Keywords Bose-Einstein condensation, magnons, superfluid ${ }^{3} \mathrm{He}$

Abstract The possibility of Bose-Einstein condensation of excitations has been discussed for a long time. The phenomenon of the phase-coherent precession of magnetization in superfluid ${ }^{3} \mathrm{He}$ and the related effects of spin superfluidity are based on the true Bose-Einstein condensation of magnons. Several different states of coherent precession has been observed in ${ }^{3} \mathrm{He}-\mathrm{B}$ : homogeneously precessing domain (HPD); persistent signal formed by $Q$-balls at very low temperatures; coherent precession with fractional magnetization; and two new modes of the coherent precession in compressed aerogel. In compressed aerogel the coherent precession has been also found in ${ }^{3} \mathrm{He}-\mathrm{A}$. Here we demonstrate that all these cases are examples of a Bose-Einstein condensation of magnons, with the magnon interaction term in the Gross-Pitaevskii equation being provided by different types of spin-orbit coupling in the background of the coherent precession.

PACS numbers: 67.57.Fg, 05.45.Yv, 11.27.+d

\section{Introduction}

Bose-Einstein condensation (BEC) is a phenomenon of formation of collective quantum state, in which the macroscopic number of particles is governed by a single wave function. The phenomenon of Bose-Einstein condensate was predicted by Einstein in 1925 . For a review see, for example Ref. 1 . The almost perfect BEC state was observed in ultra could atomic gases. In Bose liquids, the BEC is strongly modified by interactions, but still remains the key mechanism for the

1: MCBT, Institute Neel, CNRS/UJF, Grenoble, 38042, France

E-mail: yuriy.bunkov@grenoble.cnrs.fr

2: Low Temperature Laboratory, Helsinki University of Technology, Finland

E-mail: volovik@boojum.hut.fi

3: L.D. Landau Institute for Theoretical Physics, Moscow, Russia 
formation of a coherent quantum state in Bose systems, which exhibits the phenomenon of superfluidity characterized by non-dissipative superfluid mass currents discovered first in ${ }^{4} \mathrm{He}$ by P.L. Kapitza ${ }^{2}$. Superfluidity proved to be a more general phenomenon: superfluid mass current has been found in Fermi liquid ${ }^{3} \mathrm{He}$; superfluidity of electric charge - superconductivity - is known in metals; quantum chromodynamics is characterized by superfluidity of chiral charge; color superfluidity is discussed in quark matter and baryonic superfluidity - in neutron stars; etc. Here we discuss the magnon BEC which leads to superfluidity of spin current.

Strictly speaking, the theory of superfluidity and Bose-Einstein condensation is applicable to systems with conserved $U(1)$ charge or particle number. However, it can be extended to systems with a weakly violated conservation law. This means that it can be applicable to a system of sufficiently long-lived quasiparticles - discrete quanta of energy that can be treated as condensed matter counterpart of elementary particles. In magnetically ordered materials, the corresponding propagating excitations are magnons - quanta of spin waves. Under stationary conditions the density of thermal magnons is small, but they can be pumped by resonance radio-frequency (RF) field (magnetic resonance). One may expect that at very low temperatures, the non-equilibrium gas of magnons could live a relatively long time, sufficient for formation of coherent magnon condensate.

Recently there appeared a number of articles, where authors claimed the observation of BEC of quasiparticles: excitons $\frac{3}{\underline{\underline{ }}}$ and magnons ${ }^{4}$. According to Snoke $\mathrm{s}^{\underline{5}}$ to claim the observation of BEC one should demonstrate the spontaneous emergence of coherence. And, even better, to show the interference between two condensates. Since the spontaneous coherence has not been observed directly, the claim of observation of $\mathrm{BEC}$ in the above article is still under question.

In superfluid ${ }^{3} \mathrm{He}-\mathrm{B}$, the formation of a coherent state of magnons was discovered about 20 years ago ${ }^{6}$. In pulsed NMR experiments the spontaneous formation of domain with fully phase-coherent BEC of magnons has been observed even in the presence of inhomogeneous magnetic field. This domain was called the Homogeneously Precessing Domain (HPD). The main feature of HPD is the induction decay signal, which rings in many orders of magnitude longer, than prescribed by inhomogeneity of magnetic field. This means that spins precess NOT with a local Larmor frequency, but precess coherently with a common frequency and phase. This BEC can be also created and stabilized by continuous NMR pumping. In this case the NMR frequency plays a role of magnon chemical potential, which determines the density of magnon condensate. The interference between two condensates has also been demonstrated. It was shown that HPD exhibits all the properties of spin superfluidity (see Reviews $\frac{7.8}{}$ ). The main property is the existence of spin supercurrent, which transports the magnetization on a macroscopic distance more than $1 \mathrm{~cm}$ long. This spin supercurrent flows separately from the mass current, which is essentially different from the spin-polarized ${ }^{3} \mathrm{He}-\mathrm{A}_{1}$, where spin is transported by the mass current. Also the related phenomena have been observed: spin current Josephson effect; phase-slip processes at the critical current; and spin current vortex - a topological defect which is the analog of a quantized vortex in superfluids and of an Abrikosov vortex in superconductors; etc.

The spin-orbit coupling, which is responsible for the interaction between magnons, is relatively small in ${ }^{3} \mathrm{He}-\mathrm{B}$. As a result HPD represents almost pure BEC of magnons 9.10 . In typical ${ }^{3} \mathrm{He}$ experiments, the critical temperature of magnon con- 
densation is 3 orders of magnitude higher, than the temperature of superfluid transition; i.e. magnons undergo the condensation as soon as chemical potential and spin-orbit coupling allow for this process. The superfluid ${ }^{3} \mathrm{He}$ is a very unique complex macroscopic quantum system with broken spin, orbital and gauge symmetries, where the spin-orbit coupling due to dipole-dipole interaction between the spins of ${ }^{3} \mathrm{He}$ atoms can be varied experimentally. Under different conditions one can observe different types of the BEC of the gas of magnons in ${ }^{3} \mathrm{He}-\mathrm{B} \frac{11}{}$; non-topological solitons called $Q$-balls in high energy physics ${ }^{12}$; and also magnon $\mathrm{BEC}$ in ${ }^{3} \mathrm{He}-\mathrm{A}^{13}$.

\section{Coherent precession as magnon BEC}

As distinct from the static equilibrium magnetic states with broken symmetry, the phase-coherent precession is the dynamical state which experiences the offdiagonal long-range order:

$$
\left\langle\hat{S}_{+}\right\rangle=S_{+}=S \sin \beta e^{i \omega t+i \alpha} .
$$

Here $\hat{S}_{+}$is the operator of spin creation; $S_{+}=S_{x}+i S_{y} ; \mathbf{S}=\left(S_{x}, S_{y}, S_{z}=S \cos \beta\right)$ is the vector of spin density precessing in the applied magnetic field $\mathbf{H}=H \hat{\mathbf{z}}$; $\beta, \omega$ and $\alpha$ are correspondingly the tipping angle, frequency and the phase of precession. In the modes under discussion, the magnitude of the precessing spin $S$ equals to an equilibrium value of spin density $S=\chi H / \gamma$ in the applied field, where $\chi$ is spin susceptibility of ${ }^{3} \mathrm{He}-\mathrm{B}$, and $\gamma$ the gyromagnetic ratio of the ${ }^{3} \mathrm{He}$ atom (the coherent state with half of magnetization $S=(1 / 2) \chi H / \gamma$ has been also observed in bulk ${ }^{3} \mathrm{He}-\mathrm{B}^{14}$ ). Similar to the conventional mass superfluidity which also experiences the off-diagonal long-range order, the spin precession in Eq.(1) can be rewritten in terms of the complex scalar order parameter ${ }^{7,9,10}$

$$
\langle\hat{\Psi}\rangle=\Psi=\sqrt{2 S / \hbar} \sin \frac{\beta}{2} e^{i \omega t+i \alpha},
$$

If the spin-orbit interaction is small and its contribution to the spectrum of magnons is neglected in the main approximation (as it typically occurs in ${ }^{3} \mathrm{He}$ ), then $\hat{\Psi}$ coincides with the operator of the annihilation of magnons, with the number density of magnons being equal to condensate density:

$$
n_{M}=\left\langle\hat{\Psi}^{\dagger} \hat{\Psi}\right\rangle=|\Psi|^{2}=\frac{S-S_{z}}{\hbar} .
$$

This implies that the precessing states in superfluid ${ }^{3} \mathrm{He}$ realize the almost complete BEC of magnons. The small spin-orbit coupling produces a weak interaction between magnons and leads to the interaction term in corresponding GrossPitaevskii equation for the BEC of magnons (further we use units with $\hbar=1$ ):

$$
\begin{array}{r}
\frac{\delta F}{\delta \Psi^{*}}=0, \\
F=\int d^{3} r\left(\frac{|\nabla \Psi|^{2}}{2 m_{M}}-\mu|\Psi|^{2}+\bar{E}_{D}\left(|\Psi|^{2}\right)\right),
\end{array}
$$


Here the role of the chemical potential $\mu=\omega-\omega_{L}$ is played by the shift of the precession frequency from the Larmor value $\omega_{L}=\gamma H$; the latter may slightly depend on coordinates if the field gradient is applied. In coherent states, the precession frequency $\omega$ is the same throughout the whole sample even in the nonuniform field; it is determined by the number of magnons in BEC, $N_{M}=\int d^{3} r n_{M}$, which is conserved quantity if the dipole interaction is neglected. In the regime of continuous NMR, $\omega$ is the frequency of the applied RF field, $\omega=\omega_{\mathrm{RF}}$, and the chemical potential $\mu=\omega_{\mathrm{RF}}-\omega_{L}$ determines the magnon density. Finally, $m_{M}$ is the magnon mass; and $\bar{E}_{D}$ the dipole interaction averaged over the fast precession. The general form of $\bar{E}_{D}\left(|\Psi|^{2}\right)$ depends on the orientation of the orbital degrees of freedom described by the unit vector $\mathbf{I}$ of the orbital momentum, see Ref. .15 .

\section{Magnons BEC in bulk ${ }^{3} \mathrm{He}-\mathrm{B}$ and in non-deformed aerogel}

In the coherent precession in bulk ${ }^{3} \mathrm{He}-\mathrm{B}$ and in a non-deformed aerogel 16 , the spin-orbit coupling orients vector $\hat{\mathbf{I}}$ along the axis of precession, i.e. $\hat{\mathbf{I}} \| \mathbf{H}$. In this case the interaction term $\bar{E}_{D}$ expressed through the condensate order parameter has the form different from conventional 4-th order term in dilute gases 9 :

$$
\begin{array}{r}
\bar{E}_{D}=0,|\Psi|^{2}<\frac{5}{4} S, \\
\bar{E}_{D}=\frac{8}{15} \chi \Omega_{L}^{2}\left(\frac{|\Psi|^{2}}{S}-\frac{5}{4}\right)^{2},|\Psi|^{2}>\frac{5}{4} S .
\end{array}
$$

Here $\Omega_{L} \ll \omega_{L}$ is the Leggett frequency which characterizes the dipole interaction. If the chemical potential $\mu$ is negative, i.e. $\omega$ is less than $\omega_{L}$, the minimum of the Ginzburg-Landau (GL) energy $\bar{E}_{D}\left(|\Psi|^{2}\right)-\mu|\Psi|^{2}$ corresponds to $\Psi=0$, i.e. to the static state with non-precessing equilibrium magnetization $(\beta=0)$. For $\mu>0$ (i.e. for positive frequency shift), the profile of the (normalized) energy density is shown in Fig. 1 for several values of $\mu$ given in dimensionless units $\tilde{\mu}=\left(\omega_{\mathrm{RF}}^{2}-\omega_{L}^{2}\right) / \Omega_{L}^{2} \approx \mu /\left(\Omega_{L}^{2} / 2 \omega_{L}\right)$. The minimum of the GL energy corresponds to $|\Psi|^{2} / S=(5 / 4)+(15 / 32) \tilde{\mu}$. The consequence of the peculiar profile of the interaction term is that as distinct from the dilute gases the formation of the magnon BEC starts with the finite magnitude $|\Psi|^{2}=(5 / 4) S$. This means that the coherent precession starts with a tipping angle equal to the magic Leggett angle, $\beta=104^{\circ}$, and then the tipping angle increases with increasing frequency shift. This coherent state called the HPD persists indefinitely, if one applies a small RF field to compensate the losses of magnons caused by small spin-orbit interaction.

In conventional magnetic systems, magnetization precesses in the local field with the local frequency shift and thus experiences dephasing in the inhomogeneous field. In the case of magnon BEC, the rigidity of the order parameter (the gradient term in Eq. 5) plays an important role. The spatial dephasing leads to the gradient of chemical potential. This in turn excites the spin supercurrents, which finally equilibrate the chemical potential. In the steady state of magnon BEC the gradient of a local field is compensated by small gradient of magnon density $|\Psi|^{2}$ in such a way, that the precession frequency and its phase remain homogeneous throughout the whole sample. 


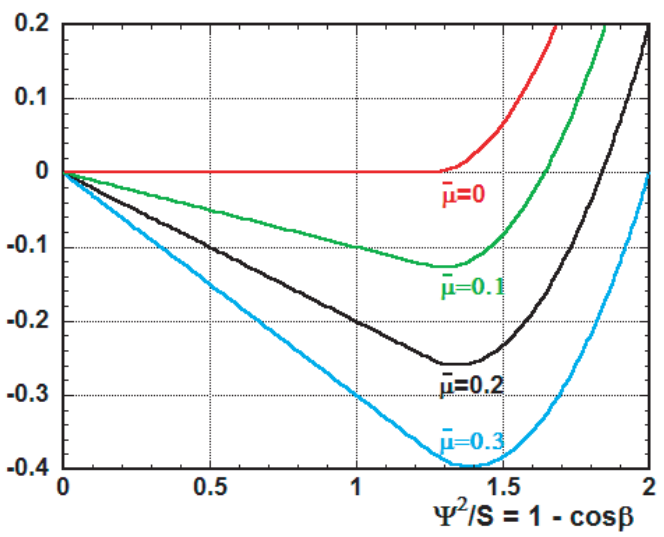

Fig. 1 (Color online) The Ginzburg-Landau energy $\bar{E}_{D}\left(|\Psi|^{2}\right)-\mu|\Psi|^{2}$ in bulk ${ }^{3} \mathrm{He}-\mathrm{B}$ as a function of magnon density (tipping angle of precession) for different values of the normalized chemical potential $\tilde{\mu}=\left(\omega_{\mathrm{RF}}^{2}-\omega_{L}^{2}\right) / \Omega_{L}^{2}$, Energy minima correspond to magnon BEC, i.e. coherent HPD states precessing with frequency shift equal to the chemical potential

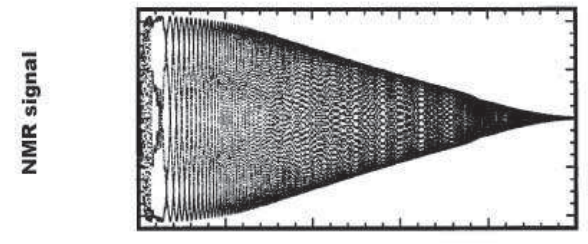

(a)

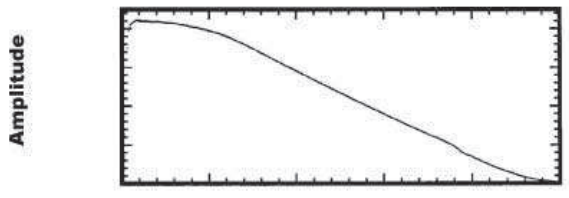

(b)

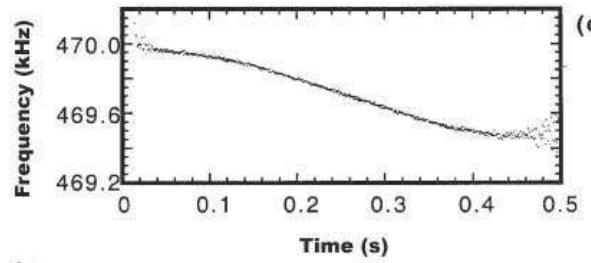

Fig. 2 (Color online) The typical signal of induction decay from the BEC of magnons; (a) stroboscopic record of the signal; (b) amplitude of signal; (c) frequency of the signal. Frequencies 469.95 and $469.4 \mathrm{kHz}$ correspond to Larmor frequency at the top and the bottom of the cell. 


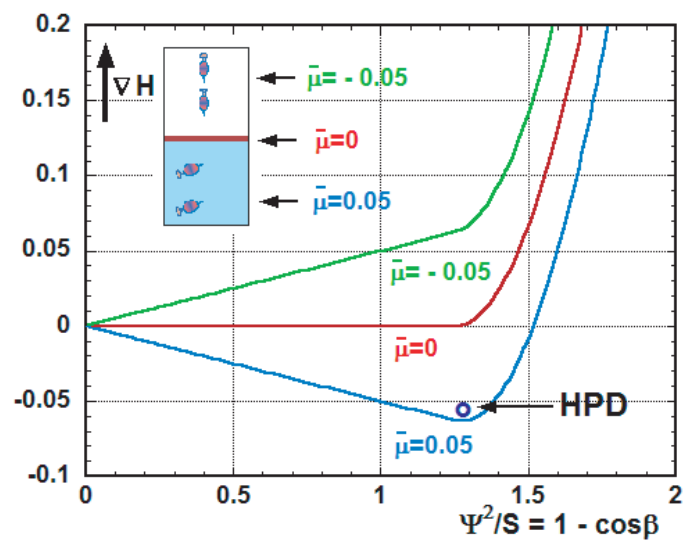

Fig. 3 (Color online) The Ginzburg-Landau energy $\bar{E}_{D}\left(|\Psi|^{2}\right)-\mu|\Psi|^{2}$ as a function of magnon density for the same frequency $\omega$, but at different Larmor frequency $\omega_{L}$ at the top and the bottom of the cell (this corresponds to different normalized chemical potential $\tilde{\mu}=\left(\omega^{2}-\omega_{L}^{2}\right) / \Omega_{L}^{2}$ )

In a pulsed NMR experiment, the magnetization is deflected by a strong RF pulse. The typical induction signal after the pulse in the cell with a large gradient of magnetic field along the axis of the cell is shown in Fig. 2. Due to the field gradient the induction signal should dephase and disappear in about $10 \mathrm{~ms}$. Instead, after a transient process of about $2 \mathrm{~ms}$, the induction signal acquires an amplitude corresponding to a $100 \%$ coherent precession of the deflected magnetization with the spontaneously emerging phase. This coherent state lives 500 times longer than the dephasing time caused by inhomogeneity.

What happens with the magnon system during the transient period? The GL energy $\bar{E}_{D}\left(|\Psi|^{2}\right)-\mu|\Psi|^{2}$ in the presence of the gradient of magnetic field is shown in Fig. 3. As soon, as at the higher field end of the cell the deflection of magnetization becomes smaller than $104^{\circ}$, the spin-orbit interaction cannot anymore compensate the gradient of magnetic field. The cell splits into two domains, in one of them the magnetization is stationary, while in other one magnons condense with the density close to $|\Psi|^{2}=(5 / 4) S$, i.e. the magnetization precesses with $\beta$ slightly above $104^{\circ}$. In the subsequent process of relaxation caused by the non-conservation of magnon number, the volume of the BEC condensate (HPD) decreases. During the relaxation, the BEC does not loose the phase coherence, but its chemical potential (precession frequency) changes. The frequency of HPD corresponds to Larmor frequency at the boundary of the domain (Fig. 3) and slowly changes with relaxation as the boundary moves down (see Fig. (2). The amplitude of the signal exactly corresponds to the record of the frequency.

Of course, the HPD has been observed, studied and explained on the basis of theory of spin superfluidity and non-linear NMR long time ago ${ }^{6}$. However, the consideration of this phenomenon in terms of the magnon BEC not only demonstrates the real system with the BEC of excitations, but also allows us to simplify the problem and to study and search for the other types of the magnon BEC in ${ }^{3} \mathrm{He}$, such as $Q$-balls $\frac{12}{2} ; \mathrm{HPD}_{2}$ state found in a deformed aerogel ${ }^{11}$; coherent precession in ${ }^{3} \mathrm{He}-\mathrm{A}$ also found in the deformed aerogel 13 ; etc. 


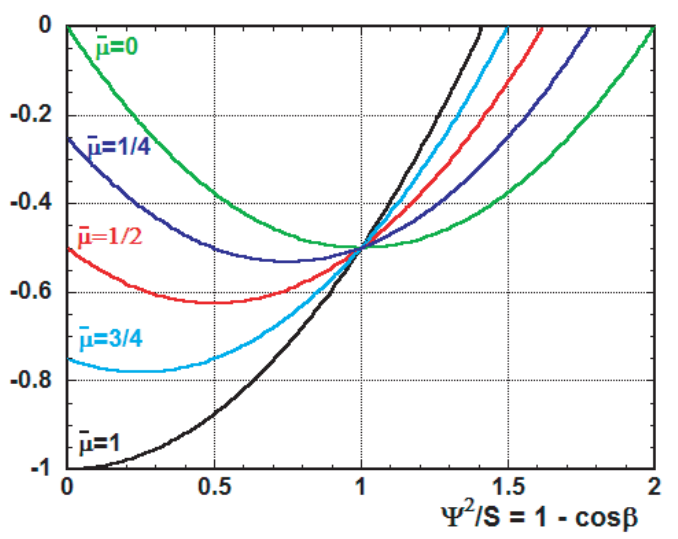

Fig. 4 (Color online) The normalized GL energy $\bar{E}_{D}\left(|\Psi|^{2}\right)-\mu|\Psi|^{2}$ in superfluid ${ }^{3} \mathrm{He}-\mathrm{A}$ in the deformed aerogel, which orients the orbital momentum î parallel to the magnetic field. The GL energy is given as a function of magnon density (tipping angle of precession) for different values of the normalized chemical potential $\tilde{\mu}=\left(\omega^{2}-\omega_{L}^{2}\right) / \Omega_{L}^{2}$. The minima of GL energy correspond to the stable magnon BEC

\section{Magnons BEC in superfluid ${ }^{3} \mathrm{He}$ in aerogel}

In superfluid ${ }^{3} \mathrm{He}-\mathrm{A}$, the spin-orbit interaction depends on the polar angle $\beta_{L}$ of the orbital vector $\mathbf{I}$ in the following way:

$$
\bar{E}_{D}=\frac{\chi \Omega_{L}^{2}}{4}\left[-2 \frac{|\Psi|^{2}}{S}+\frac{|\Psi|^{4}}{S^{2}}+\left(-2+4 \frac{|\Psi|^{2}}{S}-\frac{7}{4} \frac{|\Psi|^{4}}{S^{2}}\right) \sin ^{2} \beta_{L}\right] .
$$

While the sign of the quadratic term in Eq. (8) is not important because this term can be compensated by the shift of the chemical potential $\mu$ in Eq. 5, the sign of the quartic term is crucial for the stability of the BEC. In the static state (i.e. $\Psi=0$ ) of a bulk ${ }^{3} \mathrm{He}-\mathrm{A}$, the dipole energy is minimized when $\mathbf{I}$ is perpendicular to the magnetic field, $\sin \beta_{L}=1$. In NMR, this orientation of Î remains frozen, and the quartic term remains negative. This attractive interaction between magnons destabilizes the BEC, which means that homogeneous precession of magnetization in ${ }^{3} \mathrm{He}-\mathrm{A}$ is unstable, as was predicted by Fomin 17 and observed experimentally 18 .

Recently, we found the method, how to reorient the orbital momentum î. For that we immersed ${ }^{3} \mathrm{He}$ inside the squeezed aerogel. Even a small deformation of a few percent fixes the orbital momentum along the deformation. ${ }^{19}$. By applying the deformation along the magnetic field we fixed $\beta_{L}=0$, which made positive the quartic term in Eq. (8). As a results magnons can condense into the stable BEC state, as we observed experimentally $\underline{13}$. For $\beta_{L}=0$, the GL energy $\bar{E}_{D}\left(|\Psi|^{2}\right)-$ $\mu|\Psi|^{2}$ (Fig. 4) is similar to the traditional GL energy for the conventional BEC in atomic gases. This means that, as distinct from the HPD in ${ }^{3} \mathrm{He}-\mathrm{B}$, the minimum of the GL energy is realized even for small magnon densities, and in the experiments made in the Institute of Solid State Physics in Kashiva, Japan, we observed the formation of BEC in ${ }^{3} \mathrm{He}-\mathrm{A}$ without the domain formation. 


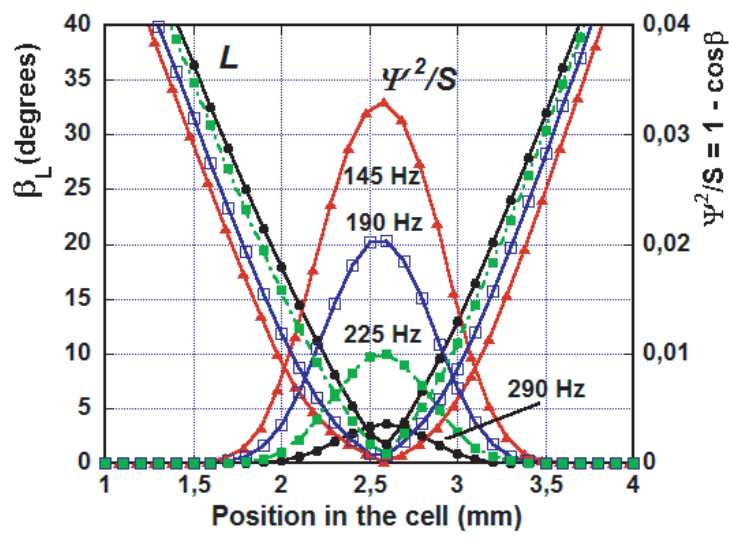

Fig. 5 (Color online) The angle of deflection of orbital momentum vector $\hat{\mathbf{l}}$ (left scale) and magnon density (right scale) as a function of position in the one dimensional cell for different total number of magnons $Q$. The NMR radiation frequency is shown for each curve.

In the 2007 experiments in Neel Institute, Grenoble we applied the aerogel deformation to reorient the orbital momentum Î in ${ }^{3} \mathrm{He}-\mathrm{B}$ perpendicular to magnetic field. For this case the shape of the GL energy allows for stable and unstable magnon BEC depending on the tipping angle $\beta$. Both BEC states were observed in pulsed NMR experiments in a good agreement with the GL energy $\underline{11}$.

\section{Magnon condensation into Q-ball}

The non-topological soliton solutions, called $Q$-balls, have been proposed by Cole$\operatorname{man}^{20}$ as a mechanism of formation of composite particles in high energy physics. $Q$-balls appear in theories containing a complex scalar field with suitable selfinteraction. They are stabilized due to the conservation of the particle number, or in general the charge $Q$. The $Q$-ball is a rather general physical object (see 21 ), which in principle can be formed in different condensed matter systems. In particular, $Q$-balls were suggested in the atomic Bose-Einstein condensates 22 .

The $Q$-balls were found in NMR experiments in superfluid ${ }^{3} \mathrm{He}-\mathrm{B}$ as a smallamplitude, but extremely long lived induction decay signal ${ }^{23}$. The role of the $Q$ charge is played by the total number of magnons $Q=\int d^{3} r n_{M} \underline{12}$. In all previous examples, magnons were almost homogeneously distributed inside the BEC state. In $Q$-balls, the condensation of magnons occurs in a local trap, produced by the texture of orbital momentum $\hat{\mathbf{I}}$. In the homogeneous state with $\mathbf{I} \| \mathbf{H}$ the spin-orbit interaction is zero for small $\Psi$ (see Eq. (6)), but in the $\hat{\mathbf{l}}$-texture $\bar{E}_{D} \neq 0$ :

$$
\bar{E}_{D}=\chi \Omega_{L}^{2}\left[\frac{4 \sin ^{2}\left(\beta_{L} / 2\right)}{5 S}|\Psi|^{2}-\frac{\sin ^{4}\left(\beta_{L} / 2\right)}{S^{2}}|\Psi|^{4}\right],
$$

where $\beta_{L}$ is the polar angle of the vector $\hat{\mathbf{l}}$. It contains the negative quartic term, which describes the attractive interaction between magnons. This form does not 
support the Bose condensation in bulk ${ }^{3} \mathrm{He}$, but supports formation of magnon liquid droplets. Such a droplet may form inside of the existing orbital texture trap. Also, the self-localized orbital trap can be spontaneously created by magnons, in a full analogy with $Q$-balls. The magnon liquid in the droplet and inside the $Q$-ball remains in the coherent precessing state.

The droplet or $Q$-ball is formed in places of the sample where the potential

$$
U(\mathbf{r})=\frac{4 \Omega_{L}^{2}}{5 \omega_{L}} \sin ^{2} \frac{\beta_{L}(\mathbf{r})}{2}
$$

produced by the $\mathbf{l}$-texture has a minimum. The precise form of a $Q$-ball depends on the particular texture and on the position in the container. Typically it is formed in the so-called flared-out texture realized in cylindrical cells. Far from the horizontal walls and close to the axis of the cell the angle $\beta_{L}$ linearly depends on the distance $r$ from the axis: $\beta_{L}(\mathbf{r}) \approx \kappa r$ (see review 24 ). The potential (10) for magnons is $U(r) \propto \kappa^{2} r^{2}$, similar to the harmonic trap used for confinement of dilute Bose gases. The peculiarity of the attractive quartic term in the GL functional in Eq. (9) is that it is not a constant but is $\propto \kappa^{4} r^{4}$. This stabilizes the condensate in the trap: at fixed number $Q$ of magnons the GL energy has minimum at some $Q$-dependent size of the magnon droplet, $r(Q)$. This is the main feature of $Q$-balls. Fig. 5 shows numerical simulations of the spatial distribution of magnon density inside the î texture for different $Q$ in one dimensional case. Note another feature of $Q$ balls: with increasing number $Q$ of the trapped magnons the potential well itself becomes modified by magnons and finally it is overwhelmingly determined by $Q$.

Simultanious formation of many different $Q$-balls has been observed in CW NMR 25 . They are generated by spin waves in the broad range of frequencies. They can be created in particular by topological defects at the walls of the cell. As distinct from CW NMR, where the $Q$-balls are generated starting continuously from $Q=0$, in pulsed NMR a $Q$-ball is formed after a large $Q$ is pumped into the cell. In this case the $3 \mathrm{D} Q$-ball is often formed on the axis of the flared-out texture, away from the horizontal walls ${ }^{26}$. Finally the lowest energy $Q$-ball collects all the magnons and radiates as a single harmonic oscillator with a slowly increasing frequency, due to the modification of the trap potential in the process of decay of the $Q$ charge, as shown in Fig. 5. The relaxation of lowest energy $Q$-balls can be compensated by additional RF pumping of magnons at any frequency above the frequency of $Q$-ball, as was found in ${ }^{27}$ and explained in 28 .

\section{Conclusion}

The variety of observed BEC states of coherent precession in superfluid ${ }^{3} \mathrm{He}$ is provided by specific interaction terms in the GL energy, which in general are different from the conventional 4-th order term in the traditional BEC. They come from the spin-orbit interaction and are governed by the orbital vector $\hat{\mathbf{l}}$. This allows us to regulate the profile of the GL energy: by applied counterflow 29 ; by deformation of aerogel $\stackrel{13,11}{2}$; by exciting the precession at one half of equilibrium magnetization ${ }^{14}$; by using flared-out 1̂-texture $\frac{12}{}$ and topological defects; etc. 
Acknowledgements We are grateful to H. Godfrin and M. Krusius for illuminating discussions. This work was done as the result of collaboration in the framework the Large Scale Installation Program ULTI of the European Union (contract number: RITA-CT-2003-505313); the project ULTIMA of "Agence Nationale de la Recherche", France (NTO5-2_41909); collaboration between CNRS and Russian Academy of Science (project 19058); and was supported in part by Russian Foundation for Basic Research (grant 06-02-16002-a).

\section{References}

1. C.J. Pethick and H. Smith, "Bose-Einstein Condensation in Dilute Gases", Ed. by Cambridge Univ. Press (2002); A.J. Leggett, Rev. Mod. Phys. 73, 307 (2001).

2. P.L. Kapitza, Nature 141, 74 (1938).

3. J.P. Eisenstein and A.H. MacDonald Nature 432, 691 (2004); J. Kasprzak et al., Nature 443, 409 (2006).

4. S.O. Democritov et al., Nature 443, 430 (2006); C. Ruegg Nature 423, 63 (2003); E. Della Torre et al., Phys. Rev. Lett., 94, 147210 (2005); T. Radu et al., Phys. Rev. Lett., 95, 127202 (2005).

5. D. Snoke et al., Nature 443, 403 (2006).

6. A.S. Borovik-Romanov et al., JETP Lett. 40, 1033 (1984); I.A. Fomin, JETP Lett. 40, 1036 (1984).

7. I.A. Fomin, Physica B 169, 153 (1991).

8. Yu.M. Bunkov, J. Low Temp. Phys. 135, 337 (2004); Spin Supercurrent and Novel Properties of NMR in ${ }^{3} \mathrm{He}$, in: Progress in Low Temp. Physics, ed. W. Halperin, Elsevier (1995), vol. 14, p. 69.

9. G.E. Volovik, cond-mat/0701180

10. Yu. M. Bunkov, J. Mag. Mag. Mat., 310, 1476 (2007).

11. J. Elbs et al., arXiv:0707.3544.

12. Yu.M. Bunkov and G.E. Volovik, Phys. Rev. Lett., 98, 265302 (2007).

13. T. Sato et al., to be published.

14. V.V. Dmitriev, et al. Phys. Rev. Lett. 78, 86 (1997).

15. Yu.M. Bunkov and G.E. Volovik, JETP, 76, 794 (1993).

16. V.V. Dmitriev et al. JETP Lett. 76, 312 (2002).

17. I.A. Fomin, JETP Lett. 39, 466 (1984).

18. A.S. Borovik-Romanov et al., JETP Lett. 39, 469 (1984).

19. T. Kunimatsu et al., JETP Lett. 86, (2007).

20. S.R. Coleman, Nucl. Phys. B 262, 263 (1985).

21. A. Kusenko, et al., Phys. Rev. Lett. 80, 3185 (1998); K. Enqvist and A. Mazumdar, Phys. Rept. 380, 99 (2003).

22. K. Enqvist and M. Laine, JCAP 0308 (2003) 003.

23. Yu.M. Bunkov et al., Phys. Rev. Lett. 69, 3092 (1992).

24. M.M. Salomaa and G.E. Volovik, Rev. Mod. Phys. 59, 533 (1987).

25. A.S. Chen et al., J. Low Temp. Phys. 110, 51 (1998).

26. D.I. Bradley et al., J. Low Temp. Phys. 134, 351 (1998).

27. D. J. Cousins et al., Phys. Rev. Lett. 82, 4484 (1999).

28. Yu.M. Bunkov, J. Low Temp. Phys. 138, 753 (2005).

29. V.V. Dmitriev et al., Phys. Rev. B 59, 165 (1999). 